\title{
Frecuencia de miopía en adultos de la Ciudad de México: resultados de una campaña de salud visual
}

\section{Frequency of myopia in adults in Mexico City: results of a visual health campaign}

\author{
Roberto Milán-Castillo*, Francisca Domínguez-Dueñas y Aleyda Molina-Lespron \\ Servicio de Oftalmología, Instituto Nacional de Rehabilitación Luis Guillermo Ibarra Ibarra, Ciudad de México, México
}

\section{Resumen}

Objetivo: Estimar la frecuencia de miopía en una población de adultos en la Ciudad de México. Método: Fue un estudio retrospectivo, transversal y descriptivo. Se calcularon los datos del equivalente esférico del ojo derecho de los pacientes examinados a partir de la refracción sin cicloplejia, determinada por medio de un autoqueratorrefractómetro durante la campaña de salud ocular que se llevó a cabo en el Instituto Nacional de Rehabilitación Luis Guillermo Ibarra Ibarra de enero a marzo de 2018. Resultados: La frecuencia de miopía encontrada en los 449 sujetos analizados fue del $45.21 \%$, tomando como punto de corte un equivalente esférico $\leq-0.50$ dioptrías; de ellos, el $39.43 \%$ tuvieron miopía leve (> -6.00 dioptrías) y el $5.79 \%$ miopía alta ( $\leq-6.00$ dioptrías de equivalente esférico). Por otra parte, solo 4 de los 449 pacientes examinados presentaron datos clínicos de miopía patológica, conformando el $0.8 \%$ de la muestra total. De los participantes restantes el $28.73 \%$ fueron clasificados como emétropes y el $26.05 \%$ como hipermétropes con un equivalente esférico $\geq 0.50$ dioptrías. Conclusiones: La frecuencia de miopía encontrada en nuestro estudio es alta y se asemeja a la de reportes previos en población mexicana infantil y de adultos jóvenes, así como a la de algunas naciones asiáticas donde la miopía es un problema de salud pública.

Palabras clave: Miopía. Error refractivo. Frecuencia. Ciudad de México. Adultos.

\section{Abstract}

Purpose: To estimate the frequency of myopia in an adult population in Mexico City. Methods: Retrospective, cross-sectional and descriptive study. The spherical equivalent of the right eye was calculated from the refraction without cycloplegia obtained with an auto kerato-refractometer in patients examined during an ophthalmic health campaign carried out at the Instituto Nacional de Rehabilitación "Luis Guillermo Ibarra Ibarra" from January to March 2018. Results: We found a frequency of myopia of $45.21 \%$ with a cut-off value of $\leq-0.5$ diopters of spherical equivalent in the 449 participants. Of these, $39.43 \%$ had mild myopia > -6.00 diopters, and 5.79\% had high myopia $\leq-6.00$ diopters of spherical equivalent. On the other hand, only 4 of the 449 examined patients had clinical signs of pathological myopia, making up $0.8 \%$ of the total sample. Of the remaining participants, $28.73 \%$ were classified as emmetropic and $26.05 \%$ as hyperopic, with a spherical equivalent $\geq 0.50$ diopters. Conclusions: The frequency of myopia observed in our study is high and similar to that informed in previous reports in Mexican children and young adults, just as in some nations in Asia where myopia is a public health problem.

Keywords: Myopia. Refractive error. Frequency. Mexico City. Adults.

Correspondencia:

*Roberto Milán-Castillo

Avda. Forestal 127, Dpto. 24a , Col. Huipulco, $\quad$ Fecha de recepción: 19-11-2020 Del. Tlalpan C.P. 14370, Ciudad de México, México E-mail: robbiemilan90@gmail.com

DOI: 10.24875/RMO.M21000185 0187-4519/@ 2021 Sociedad Mexicana de Oftalmología. Publicado por Permanyer. Este es un artículo open access bajo la licencia CC BY-NC-ND (http://creativecommons.org/licenses/by-nc-nd/4.0/).
Fecha de aceptación: 07-06-2021
Disponible en internet: 01-09-2021 Rev Mex Oftalmol. 2021;95(5):203-208

www.rmo.com.mx 


\section{Introducción}

La miopía se define como un error refractivo en el cual los rayos de luz paralelos al eje óptico que ingresan al ojo son enfocados por delante de la retina cuando la acomodación ocular se encuentra relajada'. La miopía representa un factor de riesgo importante para una serie de patologías oculares, como catarata nuclear $^{2}$, glaucoma ${ }^{3-5}$, desprendimiento de retina ${ }^{6}$, neovascularización coroidea y degeneración macular miópica?.

Los defectos refractivos no corregidos son considerados la primera causa mundial de discapacidad visual $^{8}$. En 2010 se estimó que la miopía afectaba a 1893 millones de personas en el mundo y, si las tasas de prevalencia actuales no cambian, las proyecciones muestran que afectará a 4,949 millones de personas (lo que representa el $49.8 \%$ de la población mundial) para el año $2050^{9}$.

La prevalencia de la miopía varía significativamente entre los diferentes grupos raciales. De acuerdo con estudios publicados, la prevalencia más alta se encuentra en Asia del este, donde se reportaron cifras de miopía del $96.5 \%{ }^{10}$ y el $83.3 \%{ }^{11}$ en individuos surcoreanos de 19 años que residían en una población urbana y rural, respectivamente, mientras que en Taiwán se estimó una prevalencia del $86.1 \%$ en adultos jóvenes entre 18 y 24 años $^{12}$, y en China fue del $84.6 \%$ en adolescentes de 17 años $^{13}$.

En México, los estudios realizados han evaluado principalmente la prevalencia de miopía en población infantil y adolescente, solamente en uno se incluyó también población adulta. En 2003 se reportó una prevalencia de miopía del 44\% en niños entre 12 y 13 años en Monterrey, Nuevo León ${ }^{14}$. En una zona suburbana del Estado de México se estudiaron niños entre 6 y 15 años, encontrando una prevalencia de miopía del $33 \%{ }^{15}$, mientras que en otra población infantil entre 6 y 12 años de una zona rural del mismo Estado se reportó una prevalencia de miopía del $9.7 \%{ }^{16}$. Finalmente, en 2017, se publicó un estudio de la proporción de los errores refractivos en 14 entidades de México en individuos de 6-90 años, encontrando un $24.8 \%$ de sujetos con miopía ${ }^{17}$.

Teniendo en cuenta las complicaciones de la miopía y otras patologías graves asociadas con la enfermedad, la miopía es un asunto relevante de salud pública ${ }^{8}$, con importantes implicaciones socioeconómicas no solo en México, sino en todo el mundo.

Hasta el momento existen escasos datos epidemiológicos en la literatura acerca de la miopía en México, por ello el objetivo del estudio fue determinar la frecuencia de esta ametropía en una población adulta de la Ciudad de México.

\section{Método}

\section{Diseño del estudio, población y muestreo}

Mediante un estudio transversal y descriptivo, se obtuvieron los datos clínicos de una población de individuos mayores de 18 años, mexicanos, que participaron en la campaña de salud ocular que se llevó a cabo en el Instituto Nacional de Rehabilitación Luis Guillermo Ibarra Ibarra de enero a marzo de 2018.

\section{Criterios de inclusión, exclusión y eliminación}

Se incluyeron sujetos mayores de 18 años, de nacionalidad mexicana, de cualquier sexo, que participaron en la campaña de salud ocular que se llevó a cabo en el Instituto Nacional de Rehabilitación Luis Guillermo Ibarra Ibarra de enero a marzo de 2018. Se excluyeron individuos que se hubieran sometido a cirugía refractiva, de catarata o vitreorretiniana. Se eliminó a los pacientes que tuvieron expediente incompleto, que no completaron los estudios del abordaje diagnóstico y aquellos con patología ocular que no permitiera obtener la refracción mediante el autoqueratorrefractómetro.

\section{Procedimientos del estudio en la campaña de salud ocular}

Se recolectaron durante 3 meses los datos de los participantes. El equipo estuvo formado por siete médicos residentes y un médico adscrito del servicio de oftalmología. Se obtuvo el consentimiento informado para participar en el estudio y se llevaron a cabo los siguientes procedimientos: historia clínica incluyendo ficha de identificación (nombre, edad, sexo y lugar de nacimiento), interrogatorio específico para identificar antecedentes oftalmológicos y síntomas oculares, evaluación de la agudeza y capacidad visual efectuada mediante la cartilla de Snellen, refracción empleando el autoqueratorrefractómetro KR-800 (Topcon, Tokio, Japón) previo a la midriasis farmacológica, biomicroscopia con lámpara de hendidura B900 (Haag-Streit AG, Köniz, Suiza), evaluación del polo posterior bajo midriasis farmacológica con un lente aéreo de 78 ० $90 \mathrm{D}$, identificando las características de la retina, la mácula y la papila óptica. 


\section{Clasificación del estado refractivo y definición de miopía patológica}

Se utilizaron datos del ojo derecho únicamente para la clasificación de los pacientes según su error refractivo, siguiendo los estándares de clasificación propuestos por el Instituto Internacional de Miopía para los estudios clínicos y epidemiológicos ${ }^{18}$. Se consideró como miopía al equivalente esférico $(E E) \leq-0.5$ dioptrías (D), miopía leve al $\mathrm{EE} \leq-0.5 \mathrm{y}>-6.00 \mathrm{D}$, miopía alta al $E E \leq-6.00 \mathrm{D}$, emetropía al $\mathrm{EE}>-0.5 \mathrm{y}<+0.5 \mathrm{D}$, e hipermetropía al EE $\geq+0.5 \mathrm{D}$. De igual forma, el Instituto Internacional de Miopía propone el término «miopía patológica» para definir la elongación axial excesiva asociada a la miopía que lleva a cambios estructurales del segmento posterior del ojo (incluyendo el estafiloma posterior, maculopatía miópica y neuropatía óptica asociada a miopía) y que puede generar una pérdida de la agudeza visual mejor corregida.

El protocolo estuvo apegado a la Declaración de Helsinki y fue aprobado por el Comité de Ética del Instituto Nacional de Rehabilitación Luis Guillermo Ibarra Ibarra. Todos los pacientes estuvieron de acuerdo y firmaron un consentimiento informado antes de ser evaluados.

\section{Resultados}

Se incluyeron 449 ojos derechos de 449 pacientes mexicanos, 304 de sexo femenino $(67.70 \%)$ y 145 de sexo masculino (32.30\%). La edad mínima fue de 21 años y la máxima de 89, con una media de 55.3 años. Las características demográficas de los participantes se resumen en la tabla 1.

Respecto a la frecuencia de miopía en los 449 pacientes, $203(45.21 \%)$ se encontraban dentro del rango de miopía (EE: $\leq-0.5 \mathrm{D}$ ); de los sujetos restantes, $129(28.73 \%)$ se consideraban emétropes (EE: > -0.5 D y < +0.5 D) y $117(26.05 \%)$ se incluyeron dentro del grupo de hipermetropía (Tabla 2). La distribución de los errores refractivos en la población total se muestra en la figura 1.

De los 203 pacientes con miopía (45.21\%), $177(39.42 \%)$ tuvieron un $\mathrm{EE} \leq-0.5 \mathrm{D}$, pero $>-6.00$ $\mathrm{D}$, categorizándolos como miopes leves; los 26 (5.79\%) pacientes restantes presentaron un $\mathrm{EE} \leq-6.00 \mathrm{D}$, por lo que se clasificaron dentro del grupo de pacientes con miopía alta (Tabla 2).

Se calcularon también los porcentajes de miopía en la población considerando puntos de corte del EE más
Tabla 1. Características clínicas y demográficas de los participantes

\begin{tabular}{|l|c|}
\hline Características & \\
\hline N. ${ }^{\circ}$ de ojos (pacientes) & $449(449)$ \\
\hline $\begin{array}{l}\text { Edad (años) } \\
\text { Media } \pm \text { DE } \\
\text { Rango }\end{array}$ & $55.3 \pm 11.5$ \\
\hline Sexo, $n$ (\%) & $21-89$ \\
\hline Femenino & \\
\hline Masculino & $304(67.71)$ \\
\hline Origen, n (\%) & $26(32.29)$ \\
\hline Ciudad de México & \\
\hline Estado de México & $348(77.51)$ \\
\hline Oaxaca & $16(3.56)$ \\
Veracruz & $11(2.45)$ \\
\hline Michoacán & $11(2.45)$ \\
Otros & $10(2.23)$ \\
\hline Enfermedades sistémicas, $n(\%)$ & $53(11.80)$ \\
\hline Hipertensión arterial & \\
\hline Diabetes mellitus & $106(23.61)$ \\
\hline Otras & $82(18.26)$ \\
\hline
\end{tabular}

DE: desviación estándar.

Tabla 2. Clasificación de los errores refractivos

\begin{tabular}{|l|c|}
\hline Error refractivo & n (\%) \\
\hline Miopía & $203(45.21)$ \\
\hline Miopía leve & $177(39.42)$ \\
\hline Miopía alta & $26(5.79)$ \\
\hline Emetropía & $129(28.73)$ \\
\hline Hipermetropía & $117(26.05)$ \\
\hline Total & $449(100)$ \\
\hline
\end{tabular}

negativos o miópicos, obteniéndose un $38.97 \%$ con un $\mathrm{EE} \leq-0.75$ y un $34.07 \%$ con un $\mathrm{EE} \leq-1.00 \mathrm{D}$.

En lo concerniente a la miopía patológica, solo $4(0.8 \%)$ de los 449 individuos examinados presentaron datos clínicos de esta alteración.

\section{Discusión}

Los estudios de frecuencia y prevalencia de miopía en México son escasos y, basados en la literatura, este es el primer estudio de frecuencia de miopía en población mexicana realizado específicamente en adultos (Tabla 3). La frecuencia del $45.21 \%$ estimada en nuestro estudio es equiparable a la proporción del $43.4 \%$ encontrada por Gómez-Salazar, et al. ${ }^{17}$ en el grupo de 20-29 
Rev Mex Oftalmol. 2021;95(5)

Tabla 3. Estudios de frecuencia o prevalencia de miopía en México

\begin{tabular}{|c|c|c|c|c|c|c|c|c|}
\hline Estudio & $\begin{array}{l}\text { Año de } \\
\text { estudio }\end{array}$ & Lugar & Zona & $n$ & $\begin{array}{l}\text { Edad } \\
\text { (años) }\end{array}$ & Cicloplejia & $\begin{array}{l}\text { Equivalente } \\
\text { esférico }\end{array}$ & $\begin{array}{c}\text { Pacientes } \\
\text { con } \\
\text { miopía }\end{array}$ \\
\hline Villarreal, et al. ${ }^{14}$ & 1999 & Monterrey, N.L. & Urbana & 1035 & $12-13$ & Sí & $\leq-0.50 \mathrm{D}$ & $44 \% *$ \\
\hline $\begin{array}{l}\text { Rodríguez-Ábrego, } \\
\text { et al. } .^{15}\end{array}$ & 2001 & $\begin{array}{l}\text { Nezahualcóyotl, } \\
\text { Méx. }\end{array}$ & Suburbana & 1136 & $6-15$ & No & $\leq-0.50 \mathrm{D}$ & $33 \% *$ \\
\hline $\begin{array}{l}\text { Gómez-Salazar, } \\
\text { et al. }{ }^{17}\end{array}$ & 2014-2015 & Varios & Varias & 676,856 & 6-90 & No & $<-0.50 \mathrm{D}$ & $24.8 \%^{\dagger}$ \\
\hline $\begin{array}{l}\text { García-Liévanos, } \\
\text { et al. }{ }^{16}\end{array}$ & $2016^{\ddagger}$ & $\begin{array}{l}\text { Temascalapa, } \\
\text { Méx. }\end{array}$ & Rural & 317 & $6-12$ & No & $\leq-0.50 \mathrm{D}$ & $9.7 \% *$ \\
\hline Milán, et al. & 2018 & $\begin{array}{l}\text { Ciudad de } \\
\text { México, Méx. }\end{array}$ & Urbana & 449 & $21-89$ & No & $\leq-0.50 \mathrm{D}$ & $45.21 \%^{\dagger}$ \\
\hline
\end{tabular}

* Estudios de prevalencia.

† Estudio de frecuencia o proporción.

${ }^{\ddagger}$ Año de publicación, no se menciona en el artículo el año en el que se realizó la obtención de los datos.

D: dioptrías.

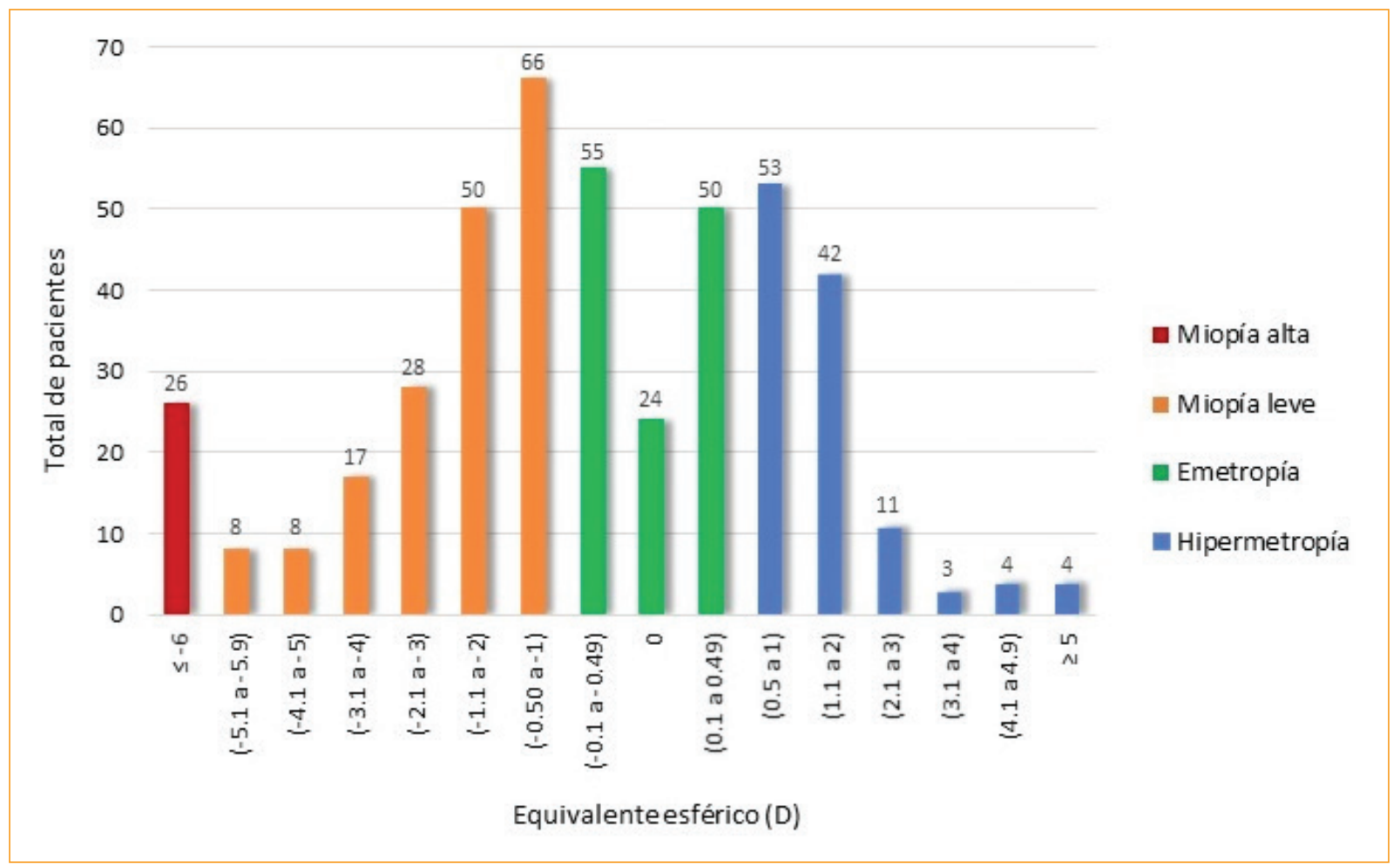

Figura 1. Distribución del error refractivo en la población total $(n=449)$ de acuerdo con el equivalente esférico obtenido. D: dioptrías.

años; sin embargo, es muy superior al $19.7 \%$, el $10.7 \%$ y el $8.9 \%$ reportados por los mismos autores en los grupos de 40-49, 50-59 y 60-69 años, respectivamente, rango de edad en el que se encontraba la gran mayoría de los participantes de nuestra campaña. No obstante, cabe destacar que estos autores designaron como miopía un $E E \leq-0.75 \mathrm{D}$. Nuestros datos también se equiparan al 44\% encontrado por Villarreal, et al. ${ }^{16}$ en 2003 en una población pediátrica en condiciones de cicloplejia y tomando como punto de corte un $E E \leq-0.5 \mathrm{D}$. 
Nuestras cifras son inferiores al compararlas con otros análisis existentes en población latina adulta, como el MIOPUR (Determinación de la Prevalencia de Miopía y su Asociación con Influencias Medioambientales en Población Colombiana Urbana y Rural), que reportó una prevalencia del $15.7 \%$ y del $9.2 \%$ en población urbana y rural, respectivamente ${ }^{19}$. De igual forma, el Los Angeles Latino Eye Study encontró una prevalencia de miopía del $16.8 \%$ en adultos mayores de 40 años de una población latina residente en los Estados Unidos de América; sin embargo, en esta publicación se tomó un $\mathrm{EE} \leq-1.00 \mathrm{D}$ para clasificar a la población dentro del grupo con dicho error refractivo ${ }^{20}$.

En comparación con estudios realizados en Asia, nuestros porcentajes fueron ligeramente inferiores a lo estimado por el Nagahama Study en Japón, con una prevalencia de miopía en adultos de 34 a 80 años del $49.97 \%{ }^{21}$, mientras que en adultos de $20-40$ años en Arabia Saudita se reportó un $48.7 \% \%^{22}$, ambos con un $\mathrm{EE} \leq-0.50 \mathrm{D}$ como definición de miopía.

En Europa se estimó una prevalencia general del $30.6 \%$ en adultos de 25 a 89 años de acuerdo con un metaanálisis en el que se incluyeron 15 estudios realizados en diversos países ${ }^{23}$.

En nuestro estudio, cuando se realizaron estimaciones adicionales de la frecuencia total mediante el ajuste del EE, se obtuvieron frecuencias que también pueden considerarse elevadas: con un punto de corte del $E E \leq-0.75 \mathrm{D}$ se obtuvo una cifra del $38.97 \%$, y con un $\mathrm{EE} \leq-1.00 \mathrm{D}$ se calculó un $34.07 \%$. El hecho de realizar estos ajustes permite que los sesgos posibles, inherentes al no haber realizado la refracción bajo cicloplejia, sean compensados en mayor medida. Fotouhi, et al. ${ }^{24}$ señalan que, en general, la sensibilidad en la obtención del error refractivo con el autorrefractómetro sin aplicación de ciclopléjicos para el diagnóstico de miopía es del $99 \%$, pero la especificidad es del $80.4 \%$; caso contrario a lo que sucede con la hipermetropía, en la cual la sensibilidad es de tan solo el $47.9 \%$, pero la especificidad es del $99.4 \%$. Se menciona además que, con este método y sin cicloplejia, a cualquier edad se sobreestima la miopía y se subestima la hipermetropía, aunque dicha sobreestimación es mayor en los grupos de 21-30 años y 31-40 años.

Los puntos de corte del EE para la clasificación del error refractivo fueron elegidos porque la mayoría de los estudios mundiales de prevalencia de miopía emplean dichas cifras, resaltando que la estandarización en las definiciones y los puntos de corte son esenciales para facilitar el abordaje basado en evidencias del estudio y manejo de la miopía, si bien también señalan que en aquellos estudios en los que no se empleen ciclopléjicos y se analice a una población más joven deberían considerarse umbrales más miópicos ${ }^{18}$.

Respecto a las cifras de miopía alta obtenidas, con un total de 26 pacientes que dieron como resultado un $5.79 \%$ de todos los pacientes, los datos son discretamente inferiores a los reportados por el Nagahama Study, en los que se encontró una prevalencia de casi el $7.89 \%$, empleando un $E E \leq-6.00 D^{21}$.

Finalmente, el porcentaje del $0.8 \%$ de miopía patológica fue inferior en comparación con lo publicado en estudios poblaciones, como el Beijing Eye Study ${ }^{25}$ en China y el Blue Mountains Study ${ }^{26}$ en Australia, con prevalencias del $3.1 \%$ y el $1.2 \%$, respectivamente.

Como mencionamos previamente, una de las limitaciones del estudio fue el no haber realizado la refracción bajo cicloplejia, si bien solo una pequeña parte de los individuos estudiados eran menores de 40 años. Asimismo, debido a la mayor concentración de participantes entre 40 y 70 años, no nos fue posible evaluar la tendencia reportada en varias publicaciones de una mayor miopía en los adultos jóvenes, seguido de reducción de la miopía en edades más avanzadas y finalmente un nuevo repunte alrededor de los 70 años $^{17,19,23}$. Por otro lado, muchos de los adultos que acudieron a la campaña de salud visual para realizarse el examen oftalmológico completo gratuito ya habían tenido contacto previo con oftalmólogos u optometristas, circunstancia que podría haber generado un mayor interés por acudir a una nueva revisión en comparación con aquel sector de la población que nunca ha requerido atención oftalmológica ni son usuarios de lentes, sobreestimando tal vez los datos obtenidos al momento de extrapolarlos a una población más amplia.

\section{Conclusiones}

La frecuencia de miopía encontrada en nuestro estudio es alta, muy superior a la prevalencia reportada en población latina de otros países; sin embargo, es semejante a la de otras publicaciones en población mexicana infantil y de adultos jóvenes, así como a la de algunas naciones asiáticas donde la miopía es un problema de salud pública. De acuerdo con esto y con la tendencia observada en los últimos años, en la que se espera que la miopía siga aumentando, consideramos conveniente que se aborde en México este tema con mayor atención, con la finalidad de poder intervenir de manera oportuna en la aparición o progresión de esta 
enfermedad de muy fácil diagnóstico, pero con repercusiones potencialmente devastadoras para la visión.

\section{Financiación}

Los autores no recibieron patrocinio para llevar a cabo este artículo.

\section{Conflicto de intereses}

Los autores declaran no tener ningún conflicto de intereses.

\section{Responsabilidades éticas}

Protección de personas y animales. Los autores declaran que para esta investigación no se han realizado experimentos en seres humanos ni en animales.

Confidencialidad de los datos. Los autores declaran que han seguido los protocolos de su centro de trabajo sobre la publicación de datos de pacientes.

Derecho a la privacidad y consentimiento informado. Los autores han obtenido el consentimiento informado de los pacientes y/o sujetos referidos en el artículo. Este documento obra en poder del autor de correspondencia.

\section{Bibliografía}

1. Németh J, Tapasztó B, Aclimandos WA, Kestelyn P, Jonas JB, de FAber JTHN, et al. Update and guidance on management of myopia. European Society of Ophthalmology in cooperation with International Myopia Institute. Eur J Ophthalmol. 2021 Mar 5;1120672121998960. doi 10.1177/1120672121998960. Online ahead of print.

2. Pan CW, Cheng CY, Saw SM, Wang JJ, Wong TY. Myopia and age-related cataract: a systematic review and meta-analysis. Am J Ophthalmol. 2013;156:1021-33.

3. Mitchell P, Hourihan F, Sandbach J, Wang J. The relationship between glaucoma and myopia. The Blue Mountains Eye Study. Ophthalmol. 1999;106:2010-15.

4. Qiu M, Wang SY, Singh K, Lin SC. Association between myopia and glaucoma in the United States Population. Invest Ophthalmol Vis Sci. 2013;54:830-5.

5. Marcus MW, de Vries MM, Junoy Montolio FG, Jansonius NM. Myopia as a risk factor for open-angle glaucoma: a systematic review and meta-analysis. Ophthalmol. 2011;118:1989-94.
6. Han X, Ong JS, An J, Craig JE, Gharahkhani P, Hewitt AW, et al. Association of myopia and intraocular pressure with retinal detachment in European descent participants of the UK Biobank Cohort: a Mendelian randomization study. JAMA Ophthalmol. 2020;138:671-8.

7. Haarman AEG, Enthoven CA, Tideman JWL, Tedja MS, Verhoeven VJM, Klaver CCW. The complications of myopia: A review and meta-analysis. Invest Opthalmol Vis Sci. 2020;61:49.

8. Bourne RRA, Stevens GA, White RA, Smith JL, Flaxman SR, Price H, et al. Causes of vision loss worldwide, 1990-2010: a systematic analysis. Lancet Global Health. 2013;1:e339-49.

9. Holden BA, Fricke TR, Wilson DA, Jong M, Naidoo KS, Sankaridurg P, et al. Global prevalence of myopia and high myopia and temporal trends from 2000 through 2050. Ophthalmol. 2016;123:1036-42.

10. Jung SK, Lee JH, Kakizaki H, Jee D. Prevalence of myopia and its association with body stature and educational level in 19-year-old male conscripts in Seoul, South Korea. Invest Ophthalmol Vis Sci. 2012;53:5579-83.

11. Lee JH, Jee D, Kwon JW, Lee WK. Prevalence and risk factors for myopia in a rural Korean population. Invest Ophthalmol Vis Sci. 2013:54:5466-71.

12. Lee YY, Lo CT, Sheu SJ, Lin JL. What factors are associated with myopia in young adults? A survey study in Taiwan Military Conscripts. Invest Ophthalmol Vis Sci. 2013:54:1026-33.

13. Wu JF, Bi HS, Wang SM, Hu YY, Wu H, Sun W, et al. Refractive error, visual acuity and causes of vision loss in children in Shandong, China: the Shandong Children Eye Study. PLoS One. 2013;8:e82763.

14. Villarreal GM, Ohlsson J, Cavazos H, Mohamed JH. Prevalence of myopia among 12-to 13-year-old schoolchildren in Northern Mexico. Optom Vis Sci. 2003;80:369-73

15. Rodríguez-Ábrego G, Sotelo-Dueñas HM. Prevalencia de miopía en escolares de una zona suburbana. Rev Med Inst Mex Seguro Soc. 2009;47:39-44.

16. García-Liévanos O, Sánchez-González L, Espinosa-Cruz N, Hernández-Flores LA, Salmerón-Leal L, Torres-Rodríguez HD. Myopia in schoolchildren in a rural community in the State of Mexico, Mexico. Clin Optometry (Auckl). 2016;8:53-6.

17. Gomez-Salazar F, Campos-Romero A, Gomez-Campaña $H$, Cruz-Zamudio C, Chaidez-Felix M, Leon-Sicairos N, et al. Refractive errors among children, adolescents and adults attending eye clinics in Mexico. Int J Ophthalmol. 2017;10:796-802

18. Flitcroft DI, He M, Jonas JB, Jong M, Naidoo K, Ohno-Matsui K, et al. IMI - Defining and classifying myopia: a proposed set of standards for clinical and epidemiologic studies. Invest Ophthalmol Vis Sci. 2019:60:M20-30.

19. Galvis V, Tello A, Otero J, Serrano AA, Gómez LM, Camacho PA, et al. Prevalence of refractive errors in Colombia: MIOPUR study. $\mathrm{Br} \mathrm{J}$ Ophthalmol. 2018;102:1320-23.

20. Tarczy-Hornoch K, Ying-Lai M, Varma R, Paz SH, Abbott LV, Azen SP, et al. Myopic refractive error in adult Latinos: the Los Angeles Latino Eye Study. Invest Ophthalmol Vis Sci. 2006;47:1845-52.

21. Nakao SY, Miyake M, Hosoda Y, Nakano E, Mori Y, Takahashi A, et al. Myopia prevalence and ocular biometry features in a general Japanese population: the Nagahama Study. Ophthalmol. 2021;128:522-31.

22. Almudhaiyan $T$, Alhamzah $A$, AlShareef $M$, Alrasheed $A$, Jaffar R, Alluhidan A, et al. The prevalence of refractive errors among Saudi adults in Riyadh, Saudi Arabia. Saudi J Ophthalmol. 2020;34:30-4.

23. Williams KM, Verhoeven VJM, Cumberland P, Bertelsen G, Wolfram C, Buitendijk GH, et al. Prevalence of refractive error in Europe: the European Eye Epidemiology (E3) Consortium. Eur J Epidemiol. 2015;30:305-15.

24. Fotouhi A, Morgan IG, Iribarren R, Khabazkhoob M, Hashemi H. Validity of noncycloplegic refraction in the assessment of refractive errors: the Tehran Eye Study. Acta Ophthalmol. 2012;90:380-6.

25. Liu HH, Xu L, Wang YX, You QS, Jonas JB. Prevalence and progression of myopic retinopathy in Chinese adults: the Beijing Eye Study. Ophthalmol. 2010;117:1763-68.

26. Vongphanit J, Mitchell P, Wang JJ. Prevalence and progression of myopic retinopathy in an older population. Ophthalmol. 2002;109:704-11. 